\title{
Recursive Linear Estimation for Discrete-Time Systems in the Presence of Different Multiplicative Observation Noises
}

\author{
C. Sánchez-González and T. M. García-Muñoz \\ Departamento de Métodos Cuantitativos para la Economía y la Empresa, Facultad de Ciencias Económicas \\ y Empresariales, Universidad de Granada, Campus Universitario de Cartuja, s/n, 18011 Granada, Spain \\ Correspondence should be addressed to C. Sánchez-González, csanchez@ugr.es
}

Received 17 September 2009; Revised 11 February 2010; Accepted 21 March 2010

Academic Editor: Xue-Zhong He

Copyright (c 2010 C. Sánchez-González and T. M. García-Muñoz. This is an open access article distributed under the Creative Commons Attribution License, which permits unrestricted use, distribution, and reproduction in any medium, provided the original work is properly cited.

This paper describes a design for a least mean square error estimator in discrete time systems where the components of the state vector, in measurement equation, are corrupted by different multiplicative noises in addition to observation noise. We show how known results can be considered a particular case of the algorithm stated in this paper.

\section{Introduction}

It was back in 1960 when Kalman [1] introduced his well-known filter. Assuming that the dynamic system is described through a state space model, Kalman considers the problem of optimum linear recursive estimation. From this event much other research work was developed including different hypothesis frameworks about system noises [2-5].

In all studies above mentioned the estimated signal (state vector) in measurement equation is only corrupted by additive noise. Rajasekaran et al. [6] consider the problem of linear recursive estimation of stochastic signals in the presence of multiplicative noise in addition to measurement noise. When multiplicative noise is a Bernoulli random variable, the system is called system with uncertain observations. Hadidi and Schwartz [7] investigate for the existence of recursive least-squares state estimators, where uncertainty about observations is caused by a binary switching sequence which is specified by a conditional probability distribution and which enters the observation equation. The proposed solution is revisited by Wang [8], proposing new formulations for the optimal filter and the one-step predictor. The estimation problem about these systems has been extensively treated [9-11]. There have been other approaches as that of Zhang et al. [12], in which the authors consider 
the infinite horizon mixed $\mathrm{H} 2 / \mathrm{H} \infty$ control for discrete-time stochastic systems with state and disturbance-dependent noise. In a very recent study [13], the optimal H2 ltering problems associated respectively with possible delay of one sampling period, uncertain observations and multiple packet drop-outs are studied under a unied framework. In particular Sahebsara et al. [13] propose the following observation equation:

$$
y(t)=\xi(t) z(t)+(1-\xi(t)) y(t-1)
$$

where $z(t)$ is the m-real valued measured output, $y(t)$ is the measurement received by the estimator to be designed, and $\xi(t)$ is a white binary distributed random variable with $P\{\xi(t)=$ $1\}=\alpha(t)$ and $P\{\xi(t)=0\}=1-\alpha(t)$, where $0 \leq \alpha(t) \leq 1$ and is uncorrelated with other random variables. The model introduced by Sahebsara et al. [13] describes packet drop-outs in networked estimation. The model says that the latest measurement received will be used if the current measurement is lost. Some other authors like Nakamori [14] focus their attention on the recursive estimation technique using the covariance information in linear stationary discrete-time systems when the uncertain observations are given.

We propose in this paper a design for a least mean square error (LMSE) estimator in discrete-time systems where the components of the state vector, in measurement equation, are corrupted by different multiplicative noises in addition to observation noise. The estimation problems treated include one-stage prediction and filtering.

The presented algorithm can be considered as a general algorithm because, with particular specifications, this algorithm degenerates in known results as in Kalman [1], Rajasekaran et al. [6], Nahi [9], and Sánchez-González and García-Muñoz [11]. It can also be inferred that if multiplicative noises are Bernoulli random variables, such situation is not, properly speaking, a system with uncertain observations because the components of the state can be present in the observation with different probabilities. Therefore, the presented algorithm solves the estimation problems in this new system specification with complete uncertainty about signals.

\section{Statement and Notation}

We now introduce symbols and definitions used across the paper. Let the following linear discrete-time dynamic system with $n \times 1$ elements be the state vector $x(k)$ :

State Equation:

$$
\begin{gathered}
x(k+1)=\Phi(k+1, k) x(k)+\Gamma(k+1, k) \omega(k), \quad k \geq 0, \\
x(0)=x_{0},
\end{gathered}
$$

and $m \times 1$ observation vector $z(k)$ be given by Observation Equation:

$$
z(k)=H(k) \tilde{\gamma}(k) x(k)+v(k), \quad k \geq 0,
$$

where $\Phi(k+1, k), \Gamma(k+1, k)$, and $H(k)$ are known matrices with appropriate dimensions. 
Usual and specific hypothesis regarding probability behavior for random variables is introduced to formalize the model as follows:

(H.1) $x_{0}$ is a centered random vector with variance-covariance matrix $P(0)$,

(H.2) $\{\omega(k), k \geq 0\}$ is centered white noise with $E\left[\omega(k) \omega^{T}(k)\right]=Q(k)$.

(H.3) $\tilde{\gamma}(k)$ is a diagonal matrix

$$
\left(\begin{array}{ccc}
\gamma_{1}(k) & & \\
& \ddots & \\
& & \\
& & \gamma_{n}(k)
\end{array}\right)
$$

where $\left\{\gamma_{i}(k), k \geq 0\right\}$ is a scalar white sequence with nonzero mean $m_{i}(k)$ and variance $\sigma_{i i}(k), i=1, \ldots, n$. It is supposed that $\left\{\gamma_{i}(k), k \geq 0\right\}$ and $\left\{\gamma_{j}(k), k \geq 0\right\}$ are correlated in the same instant and $\sigma_{i j}(k)=\operatorname{Cov}\left(\gamma_{i}(k), \gamma_{j}(k)\right), i, j=1, \ldots, n$. The following matrix will be used later on:

$$
M(k)=\left(\begin{array}{ccc}
m_{1}(k) & & \\
& \ddots & \\
& & m_{n}(k)
\end{array}\right) .
$$

(H.4) $\{v(k), k \geq 0\}$ is a centered white noise sequence with variance

$$
E\left[v(k) v^{T}(k)\right]=R(k) .
$$

(H.5) $x_{0},\{\omega(k), k \geq 0\}$, and $\{v(k), k \geq 0\}$ are mutually independent.

(H.6) The sequences $\left\{\gamma_{i}(k), k \geq 0\right\}, i=1, \ldots, n$ are independent of initial state

$$
x_{0},\{\omega(k), k \geq 0\}, \quad\{v(k), k \geq 0\}
$$

As we can observe, the components of the state vector, in the observation equation, are corrupted by multiplicative noises in addition to measurement noise.

Let $\widehat{x}(k / l)$ be the LMSE estimate of $x(k)$ given observations $z(0), \ldots, z(l) . e(k / l)=$ $x(k)-\widehat{x}(k / l)$ denoting the estimation error, and the corresponding covariance matrix is $P(k / l)=\left[e(k / l) e^{T}(k / l)\right]$.

The LMSE linear filter and one-step ahead predictor of the state $x(k)$ are presented in the next section. 


\section{Prediction and Filter Algorithm}

Theorem 3.1. The one-step ahead predictor and filter are given by

$$
\begin{gathered}
\widehat{x}(k+1 / k)=\Phi(k+1, k) \widehat{x}(k / k), \quad k \geq 0, \\
\widehat{x}(0 /-1)=0, \\
\widehat{x}(k / k)=\widehat{x}(k / k-1)+F(k)[z(k)-H(k) M(k) \widehat{x}(k / k-1)], \quad k \geq 0 .
\end{gathered}
$$

The filter gain matrix verifies

$$
F(k)=P(k / k-1) M(k) H^{T}(k) \Pi^{-1}(k),
$$

where

$$
\Pi(k)=H(k) \widetilde{S}(k) H^{T}(k)+H(k) M(k) P(k / k-1) M(k) H^{T}(k)+R(k)
$$

with

$$
\begin{aligned}
& \tilde{S}(k)=\left(\begin{array}{cccc}
\sigma_{11}(k) S_{11}(k) & \sigma_{12}(k) S_{12}(k) & \cdots & \sigma_{1 n}(k) S_{1 n}(k) \\
\sigma_{12}(k) S_{21}(k) & \sigma_{22}(k) S_{22}(k) & \cdots & \sigma_{2 n}(k) S_{2 n}(k) \\
\vdots & \vdots & \ddots & \vdots \\
\sigma_{1 n}(k) S_{1 n}(k) & \sigma_{2 n}(k) S_{2 n}(k) & \cdots & \sigma_{n n}(k) S_{n n}(k)
\end{array}\right) \\
& S_{i j}(k)=I_{i} S(k) I_{j}^{T}, \quad \text { where } I_{i}=\left(\begin{array}{llllllll}
0 & 0 & \cdots & 0 & \underset{(i)}{1} & 0 & \cdots & 0
\end{array}\right)_{1 \times n}, \\
& S(k+1)=\Phi(k+1, k) S(k) \Phi^{T}(k+1, k)+\Gamma(k+1, k) Q(k) \Gamma^{T}(k+1, k), \quad k \geq 0, \\
& S(0)=P(0) \text {. }
\end{aligned}
$$

The prediction and filter error covariance matrices satisfy

$$
\begin{gathered}
P(k+1 / k)=\Phi(k+1, k) P(k / k) \Phi^{T}(k+1, k)+\Gamma(k+1, k) Q(k) \Gamma^{T}(k+1, k), \quad k \geq 0, \\
P(0 /-1)=P(0), \\
P(k / k)=P(k / k-1)-F(k) \Pi(k) F^{T}(k), \quad k \geq 0 .
\end{gathered}
$$

Proof. By the state equation it is easy to prove that the predictor $\Phi(k+1, k) \widehat{x}(k / k)$ satisfies the orthogonal projection lemma (OPL) [15]. In the initial instant, the estimate of $x(0)$ is its mean, so that $\widehat{x}(0 /-1)=0$.

As a consequence of the orthogonal projection theorem [15], the state filter can be written as a function of the one-step ahead predictor as

$$
\widehat{x}(k / k)=\widehat{x}(k / k-1)+F(k) \delta(k), \quad k \geq 0,
$$


where $\delta(k)=z(k)-\widehat{z}(k / k-1)$ is the innovation process. Its expression is obtained below.

Since $\widehat{z}(k / k-1)$ is the orthogonal projection of $z(k)$ onto the subspace generated by observations $\{z(0), \ldots, z(k-1)\}$, we know that this is the only element in that subspace verifying

$$
E\left[z(k) z^{T}(\alpha)\right]=E\left[\widehat{z}(k / k-1) z^{T}(\alpha)\right], \quad \alpha=0, \ldots, k-1
$$

Then, by the observation equation and the hypotheses (H.3)-(H.6), it can be seen that $\widehat{z}(k / k-$ $1)=H(k) M(k) \widehat{x}(k / k-1)$, and the innovation process for the problem we are solving is given by

$$
\delta(k)=z(k)-H(k) M(k) \widehat{x}(k / k-1) .
$$

To obtain the gain matrix $F(k)$, we observe that, given that the OPL holds, $E\left[e(k / k) z^{T}(k)\right]=$ 0 , and we have

$$
E\left[e(k / k-1) z^{T}(k)\right]=F(k) \Pi(k)
$$

where $\Pi(k)$ are the covariance matrices of the innovation. From the observation equation and the hypotheses (H.2)-(H.6), it can easily be checked that

$$
E\left[e(k / k-1) z^{T}(k)\right]=P(k / k-1) M(k) H^{T}(k)
$$

and therefore $F(k)=P(k / k-1) M(k) H^{T}(k) \Pi^{-1}(k)$.

To obtain the covariance matrices of the innovation process, it can be seen that

$$
\delta(k)=H(k) \tilde{\gamma}(k) x(k)+v(k)-H(k) M(k) \widehat{x}(k / k-1),
$$

and by adding and subtracting $H(k) M(k) x(k)$,

$$
\delta(k)=H(k)(\widetilde{\gamma}(k)-M(k)) x(k)+v(k)+H(k) M(k) e(k / k-1) .
$$

Then

$$
\begin{aligned}
\Pi(k)= & E\left[\delta(k) z^{T}(k)\right] \\
= & H(k) E\left[(\tilde{\gamma}(k)-M(k)) x(k) z^{T}(k)\right]+E\left[v(k) z^{T}(k)\right] \\
& +H(k) M(k) E\left[e(k / k-1) z^{T}(k)\right] .
\end{aligned}
$$


Let us work out each of the terms in previous expression. By the observation equation we have that

$$
\begin{aligned}
H(k) & E\left[(\tilde{\gamma}(k)-M(k)) x(k) z^{T}(k)\right] \\
& =H(k) E\left[(\tilde{\gamma}(k)-M(k)) x(k) x^{T}(k) \tilde{\gamma}(k)\right] H^{T}(k)+H(k) E\left[(\tilde{\gamma}(k)-M(k)) x(k) v^{T}(k)\right],
\end{aligned}
$$

and according to hypotheses in (H.4)-(H.6) the second term can be cancelled. Adding and subtracting $H(k) E\left[(\tilde{\gamma}(k)-M(k)) x(k) x^{T}(k) M(k)\right] H^{T}(k)$,

$$
\begin{aligned}
H(k) E\left[(\tilde{\gamma}(k)-M(k)) x(k) z^{T}(k)\right]= & H(k) E\left[(\tilde{\gamma}(k)-M(k)) x(k) x^{T}(k)(\tilde{\gamma}(k)-M(k))\right] H^{T}(k) \\
& +H(k) E\left[(\tilde{\gamma}(k)-M(k)) x(k) x^{T}(k)\right] M(k) H^{T}(k),
\end{aligned}
$$

where the second term is zero by (H.3) and (H.6). According to (H.6), if we label $S_{i j}(k)=$ $E\left[x_{i}(k) x_{j}(k)\right]$ for $i, j=1, \ldots, n$, we get

$$
\begin{aligned}
\widetilde{S}(k) \equiv & E\left[(\tilde{\gamma}(k)-M(k)) x(k) x^{T}(k)(\tilde{\gamma}(k)-M(k))\right] \\
& =E\left[\left(\begin{array}{c}
\left(\gamma_{1}(k)-m_{1}(k)\right) x_{1}(k) \\
\left(\gamma_{2}(k)-m_{2}(k)\right) x_{2}(k) \\
\vdots \\
\left(\gamma_{n}(k)-m_{n}(k)\right) x_{n}(k)
\end{array}\right)\left(\begin{array}{c}
\left(\gamma_{1}(k)-m_{1}(k)\right) x_{1}(k) \\
\left(\gamma_{2}(k)-m_{2}(k)\right) x_{2}(k) \\
\vdots \\
\left(\gamma_{n}(k)-m_{n}(k)\right) x_{n}(k)
\end{array}\right)\right] \\
& =\left(\begin{array}{cccc}
\sigma_{11}(k) S_{11}(k) & \sigma_{12}(k) S_{12}(k) & \cdots & \sigma_{1 n}(k) S_{1 n}(k) \\
\sigma_{12}(k) S_{12}(k) & \sigma_{22}(k) S_{22}(k) & \cdots & \sigma_{2 n}(k) S_{2 n}(k) \\
\vdots & \vdots & \ddots & \vdots \\
\sigma_{1 n}(k) S_{1 n}(k) & \sigma_{1 n}(k) S_{2 n}(k) & \cdots & \sigma_{n n}(k) S_{n n}(k)
\end{array}\right) .
\end{aligned}
$$

Therefore

$$
H(k) E\left[(\tilde{\gamma}(k)-M(k)) x(k) z^{T}(k)\right]=H(k) \tilde{S}(k) H^{T}(k) .
$$

On the other hand, by the observation equation and (H.4)-(H.6),

$$
E\left[v(k) z^{T}(k)\right]=E\left[v(k) x^{T}(k) \tilde{\gamma}(k)\right] H^{T}(k)+E\left[v(k) v^{T}(k)\right]=R(k) .
$$


By the same reasons,

$$
\begin{aligned}
H(k) M(k) E\left[e(k / k-1) z^{T}(k)\right] & =H(k) M(k) E\left[e(k / k-1) x^{T}(k)\right] M(k) H^{T}(k) \\
& =H(k) M(k) P(k / k-1) M(k) H^{T}(k) .
\end{aligned}
$$

Shortly, the covariance matrices of the innovations process verify

$$
\Pi(k)=H(k) \widetilde{S}(k) H^{T}(k)+R(k)+H(k) M(k) P(k / k-1) M(k) H^{T}(k) .
$$

To obtain the components $S_{i j}(k)$ of the $\widetilde{S}(k)$, we only need to observe that

$$
S_{i j}(k)=I_{i} S(k) I_{j}^{T}
$$

where $S(k)=E\left[x(k) x^{T}(k)\right]$ and $I_{i}=\left(\begin{array}{llllllll}0 & 0 & \cdots & 0 & 1 & 0 & \cdots & 0\end{array}\right)_{1 \times n}$. The following recursive expression of $S(k)$ is immediate given that $\{\omega(k), k \geq 0\}$ is a white noise sequence and independent of $x(0)$ :

$$
\begin{gathered}
S(k+1)=\Phi(k+1, k) S(k) \Phi^{T}(k+1, k)+\Gamma(k+1, k) Q(k) \Gamma^{T}(k+1, k), \quad k \geq 0 \\
S(0)=P(0) .
\end{gathered}
$$

The expression of the prediction error covariance matrices

$$
P(k+1 / k)=\Phi(k+1, k) P(k / k) \Phi^{T}(k+1, k)+\Gamma(k+1, k) Q(k) \Gamma^{T}(k+1, k)
$$

is immediate since $e(k+1 / k)=\Phi(k+1, k) e(k / k)+\Gamma(k+1, k) \omega(k)$.

In the other hand, given that $e(k / k)=e(k / k-1)-F(k) \delta(k)$ then

$$
\begin{aligned}
P(k / k)= & P(k / k-1)-E\left[e(k / k-1) \delta^{T}(k)\right] F^{T}(k) \\
& -F(k) E\left[\delta(k) e^{T}(k / k-1)\right]+F(k) \Pi(k) F^{T}(k)
\end{aligned}
$$

It can be observed that

$$
E\left[\delta(k) e^{T}(k / k-1)\right]=E\left[z(k) e^{T}(k / k-1)\right]-H(k) M(k) E\left[\widehat{x}(k / k-1) e^{T}(k / k-1)\right],
$$

where the second term cancels according to OPL, and by (3.9) it is obtained that

$$
E\left[\delta(k) e^{T}(k / k-1)\right]=\Pi(k) F^{T}(k)
$$

and then $P(k / k)=P(k / k-1)-F(k) \Pi(k) F^{T}(k)$. 
Next, we see how some known results can be considered as particular specifications of the general model proposed in this paper.

(i) If $\gamma_{1}(k)=\cdots=\gamma_{n}(k)=1$, the state vectors are not corrupted by a multiplicative noise, then

$$
\begin{gathered}
\tilde{\gamma}(k)=M(k)=I_{n \times n}, \\
\sigma_{i j}(k)=0, \quad \forall i, j
\end{gathered}
$$

and our algorithm degenerates in Kalman's [1] algorithm.

(ii) If $\gamma_{1}(k)=\cdots=\gamma_{n}(k)=U(k)$ where $\{U(k), k \geq 0\}$ is a scalar white sequence with nonzero mean $m(k)$ and variance $n(k)$, we end up with Rajasekaran's et al. [6] framework, $\tilde{\gamma}(k)=U(k) I_{n \times n}$, where the state vector (all components) is corrupted by multiplicative noise. In this case,

$$
\begin{gathered}
M(k)=m(k) I_{n \times n}, \\
\sigma_{i j}(k)=n(k), \quad \forall i, j
\end{gathered}
$$

and the presented algorithm collapses in Rajasekaran's.

(iii) If $\gamma_{1}(k)=\cdots=\gamma_{n}(k)=\gamma(k)$ where $\{\gamma(k), k \geq 0\}$ is a sequence of Bernoulli independent random variable with $P[\gamma(k)=1]=p(k)$, then $\tilde{\gamma}(k)=\gamma(k) I_{n \times n}$ and we end up with Nahi's [9] framework, where the state vector is present in the observation with probability $p(k)$. In this case,

$$
\begin{gathered}
M(k)=p(k) I_{n \times n,} \\
\sigma_{i j}(k)=p(k)(1-p(k)), \quad \forall i, j,
\end{gathered}
$$

and the new algorithm collapses in Nahi's one.

(iv) If $\gamma_{1}(k)=\cdots=\gamma_{p}(k)=1$ and $\gamma_{p+1}(k)=\cdots=\gamma_{n}(k)=\gamma(k)$ where $\{\gamma(k), k \geq 0\}$ is a sequence of Bernoulli independent random variable with $P[\gamma(k)=1]=p(k)$, the observations can include some elements of the state vector not ensureing the presence of the resting others (Sánchez-González and García-Muñoz [11] framework). In this case

$$
\begin{gathered}
M(k)=\left(\begin{array}{cc}
I_{p \times p} & 0_{p \times(n-p)} \\
0_{(n-p) \times p} & I_{(n-p) \times(n-p)}
\end{array}\right), \\
\sigma_{i j}(k)= \begin{cases}0, & i, j \leq p, \\
0, & i \leq p, j>p, \\
0, & i>p, j \leq p, \\
p(k)(1-p(k)), & i, j>p,\end{cases}
\end{gathered}
$$

and the new algorithm degenerates in Sanchez and García's. 


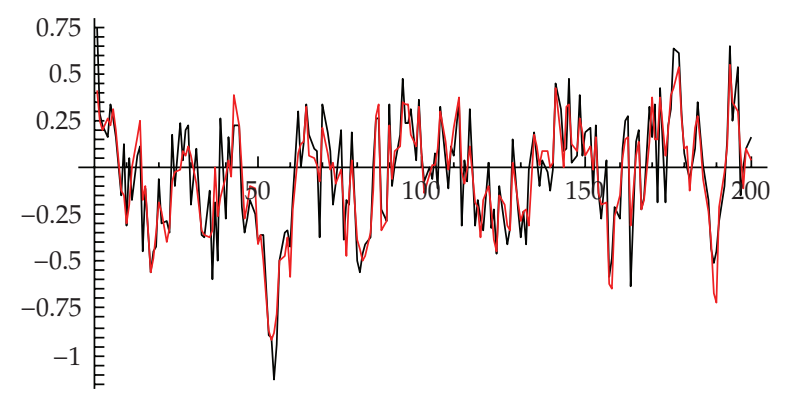

Figure 1: $x_{1}(k)$ and $\widehat{x}_{1}(k / k)$ versus $k$.

Another interesting situation appears when some of the components in the state vector are present in the observation but appear with different probabilities. Such a situation is not a system with uncertain observations. The present algorithm solves estimation problems in this type of system; it is only necessary to suppose that the multiplicative noises are different Bernoulli random variables.

\section{Some Numerical Simulation Examples}

We show now some numerical examples to illustrate the filtering and prediction algorithm presented in Theorem 3.1.

Example 4.1. We consider the following linear system described by the dynamic equation:

$$
\begin{gathered}
\left(\begin{array}{l}
x_{1}(k+1) \\
x_{2}(k+1)
\end{array}\right)=\left(\begin{array}{ll}
0.06 & 0.67 \\
0.60 & 0.23
\end{array}\right)\left(\begin{array}{l}
x_{1}(k) \\
x_{2}(k)
\end{array}\right)+\left(\begin{array}{l}
0.02 \\
0.24
\end{array}\right) \omega(k), \quad k \geq 0, \\
\left(\begin{array}{l}
x_{1}(0) \\
x_{2}(0)
\end{array}\right)=\left(\begin{array}{l}
x_{10} \\
x_{20}
\end{array}\right), \\
z(k)=\left(\begin{array}{ll}
0.85 & 0.42
\end{array}\right)\left(\begin{array}{cc}
r_{1}(k) & 0 \\
0 & \gamma_{2}(k)
\end{array}\right)\left(\begin{array}{l}
x_{1}(k) \\
x_{2}(k)
\end{array}\right)+v(k), \quad k \geq 0,
\end{gathered}
$$

where $\{\omega(k), k \geq 0\}$ is centered Gaussian white noise with $Q(k)=2.89 ; x_{10}$ and $x_{20}$ are centered Gaussian random variables with variances equal to $0.5 ;\left\{\gamma_{1}(k), k \geq 0\right\}$ and $\left\{\gamma_{2}(k), k \geq 0\right\}$ are Gaussian white noises with means 2 and 3 and variances $\sigma_{11}$ and $\sigma_{22}$, respectively; $\left\{\gamma_{1}(k), k \geq 0\right\}$ and $\left\{\gamma_{2}(k), k \geq 0\right\}$ are independent; $\{v(k), k \geq 0\}$ is centered Gaussian white noise with variance $R=0.1$.

Using the estimation algorithm of Theorem 3.1, we can calculate the filtering estimate $\hat{x}(k / k)$ of the state recursively. Figures 1 and 2 illustrate the state $x_{i}(k)$ and the filter $\widehat{x}_{i}(k / k)$, for $i=1,2$, versus $k$ for the multiplicative Gaussian observation noises $\gamma_{1} \rightarrow N(2, \sqrt{0.5})$ and $r_{2} \rightarrow N(3, \sqrt{0.1})$. The state is represented with black and the filter with red color. 
Table 1: MSV of filtering errors $x_{1}(k)-\widehat{x}_{1}(k / k), k=1,2, \ldots, 200$.

\begin{tabular}{lccc}
\hline & $\sigma_{22}=0.1$ & $\sigma_{22}=0.5$ & $\sigma_{22}=1$ \\
\hline$\sigma_{11}=0.1$ & 0.0171184 & 0.0194455 & 0.020916 \\
$\sigma_{11}=0.5$ & 0.022236 & 0.0211678 & 0.022704 \\
$\sigma_{11}=1$ & 0.0232388 & 0.023623 & 0.0237136 \\
\hline
\end{tabular}

Table 2: MSV of filtering errors $x_{2}(k)-\widehat{x}_{2}(k / k), k=1,2, \ldots, 200$.

\begin{tabular}{lccc}
\hline & $\sigma_{22}=0.1$ & $\sigma_{22}=0.5$ & $\sigma_{22}=1$ \\
\hline$\sigma_{11}=0.1$ & 0.0556318 & 0.0656372 & 0.0671069 \\
$\sigma_{11}=0.5$ & 0.0698304 & 0.0703345 & 0.0690113 \\
$\sigma_{11}=1$ & 0.0739651 & 0.075727 & 0.0730605 \\
\hline
\end{tabular}

Tables 1 and 2 show the mean-square values (MSVs) of the filtering errors $x_{i}(k)-$ $\widehat{x}_{i}(k / k)$ for $i=1,2$ and $k=1,2, \ldots, 200$ corresponding to multiplicative white observation noises:

$$
\begin{aligned}
& r_{1}: N(2, \sqrt{0.1}) ; \quad N(2, \sqrt{0.5}) ; \quad N(2, \sqrt{1}), \\
& \gamma_{2}: N(3, \sqrt{0.1}) ; \quad N(3, \sqrt{0.5}) ; \quad N(3, \sqrt{1}) .
\end{aligned}
$$

Example 4.2. We consider a linear system described by (4.1) where $\left\{\gamma_{1}(k), k \geq 0\right\}$ and $\left\{\gamma_{2}(k), k \geq 0\right\}$ are sequences of independent Bernoulli random variables being 1 with probabilities $p_{1}$ and $p_{2}$, respectively.

Figures 3 and 4 illustrate the state $x_{i}(k)$ and the filter $\widehat{x}_{i}(k / k)$, for $i=1,2$, versus $k$ for the multiplicative observation noises $\gamma_{1} \rightarrow$ Bernoulli(0.5) and $\gamma_{2} \rightarrow$ Bernoulli(1). The state is represented with red and the filter with black color.

Tables 3 and 4 show the mean-square values (MSVs) of the filtering errors $x_{i}(k)-$ $\hat{x}_{i}(k / k)$ for $i=1,2$ and $k=1,2, \ldots, 200$ corresponding to multiplicative white observation noises:

$$
\begin{array}{lll}
\gamma_{1}: \text { Bernoulli(0.1), } & \text { Bernoulli(0.5), } & \text { Bernoulli(1), } \\
\gamma_{2}: \text { Bernoulli(0.1), } & \text { Bernoulli(0.5), } & \text { Bernoulli(1). }
\end{array}
$$

As we can observe, the simulation graphs and the MSVs of the filtering in both examples show the effectiveness of the new algorithm.

\section{Conclusions}

For linear discrete-time stochastic systems where the components of the state vector are corrupted by different multiplicative noises added to the observation noises we have derived the optimal linear estimators including filter, predictor, and smoother in the minimum variance sense by applying the innovation analysis approach. Our solutions are given in 


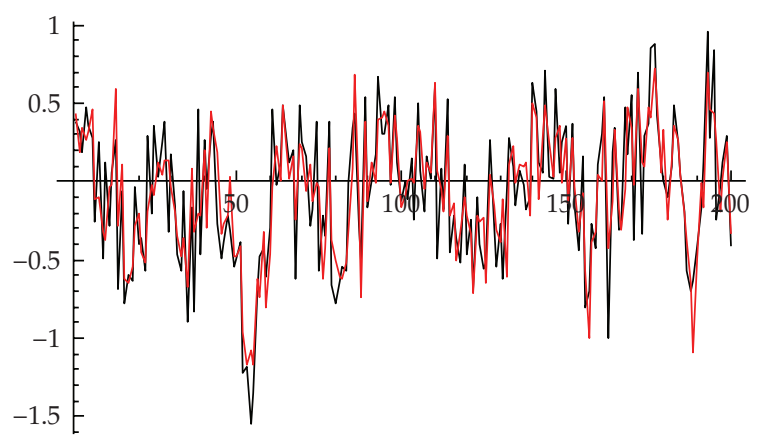

Figure 2: $x_{2}(k)$ and $\widehat{x}_{2}(k / k)$ versus $k$.

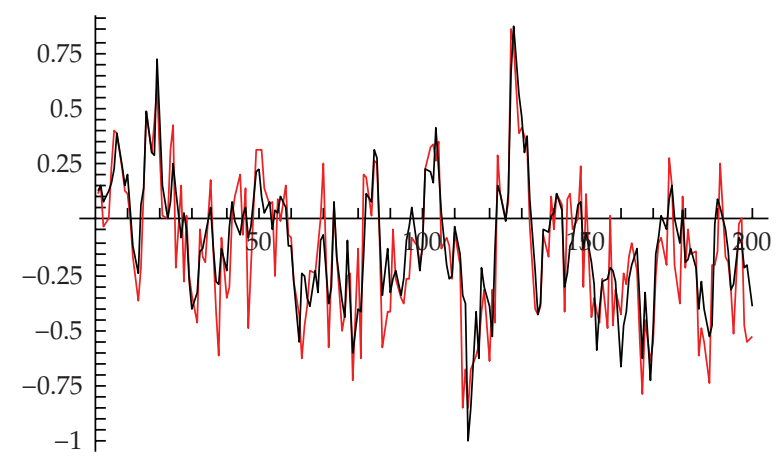

Figure 3: $x_{1}(k)$ and $\hat{x}_{1}(k / k)$ versus $k$.

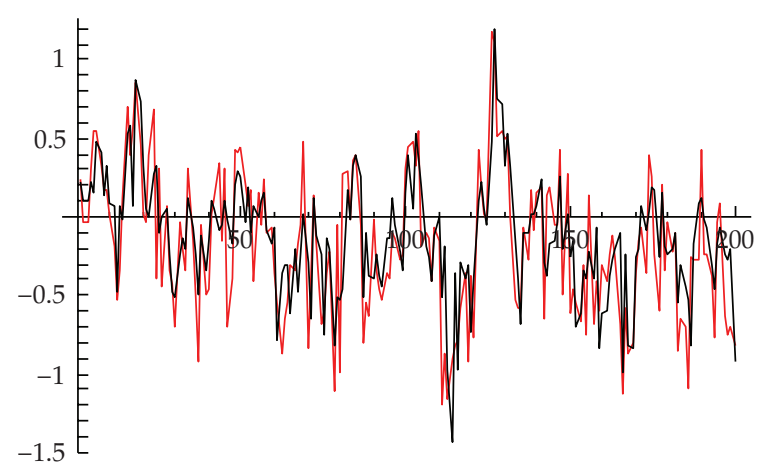

Figure 4: $x_{2}(k)$ and $\widehat{x}_{2}(k / k)$ versus $k$.

Table 3: MSV of filtering errors $x_{1}(k)-\widehat{x}_{1}(k / k), k=1,2, \ldots, 200$.

\begin{tabular}{lccc}
\hline & $p_{2}=0.1$ & $p_{2}=0.5$ & $p_{2}=1$ \\
\hline$p_{1}=0.1$ & 0.0948355 & 0.0696956 & 0.0273215 \\
$p_{1}=0.5$ & 0.0616283 & 0.049987 & 0.0333839 \\
$p_{1}=1$ & 0.013194 & 0.0197576 & 0.0154067 \\
\hline
\end{tabular}


Table 4: MSV of filtering errors $x_{2}(k)-\widehat{x}_{2}(k / k), k=1,2, \ldots, 200$.

\begin{tabular}{lccc}
\hline & $p_{2}=0.1$ & $p_{2}=0.5$ & $p_{2}=1$ \\
\hline$p_{1}=0.1$ & 0.211223 & 0.164514 & 0.0634171 \\
$p_{1}=0.5$ & 0.184817 & 0.155435 & 0.09182 \\
$p_{1}=1$ & 0.154539 & 0.130428 & 0.0708851 \\
\hline
\end{tabular}

terms of general expressions for the innovations, cevariance matrices, and gain and have the interesting fact that they result in different well-known scenarios; in particular, four of them can be considered; particularized cases of our algorithm when different values of the noise are considered, those cases are those of Kalman [1], Rajasekaran et al. [6], Nahi [9], and more recently Sánchez-González and García-Muñoz [11].

\section{References}

[1] R. E. Kalman, "A new approach to linear filtering and prediction problems," Journal of Basic Engineering, vol. 82, pp. 35-45, 1960.

[2] R. E. Kalman, "New methods in Wiener filtering theory," in Proceedings of the 1st Symposium on Engineering Application of Random Function Theory and Probability, pp. 270-387, John Wiley \& Sons, New York, NY, USA, 1963.

[3] J. S. Meditch, Stochastic Optimal Linear Estimation and Control, McGraw-Hill, New York, NY, USA, 1969.

[4] A. H. Jazwinski, Stochastic Processes and Filtering Theory, Academic Press, New York, NY, USA, 1970.

[5] A. Kowalski and D. Szynal, "Filtering in discrete-time systems with state and observation noises correlated on a finite time interval," IEEE Transactions on Automatic Control, vol. 31, no. 4, pp. 381-384, 1986.

[6] P. K. Rajasekaran, N. Satyanarayana, and M. D. Srinath, "Optimum linear estimation of stochastic signals in the presence of multiplicative noise," IEEE Transactions on Aerospace Electronic Systems, vol. 7, no. 3, pp. 462-468, 1971.

[7] M. T. Hadidi and S. C. Schwartz, "Linear recursive state estimators under uncertain observations," IEEE Transactions on Automatic Control, vol. 24, no. 6, pp. 944-948, 1979.

[8] X. D. Wang, "Recursive algorithms for linear LMSE estimators under uncertain observations," IEEE Transactions on Automatic Control, vol. 29, no. 9, pp. 853-854, 1984.

[9] N. E. Nahi, "Optimal recursive estimation with uncertain observation," IEEE Transactions on Information Theory, vol. 15, pp. 457-462, 1969.

[10] A. H. Hermoso and J. L. Pérez, "Linear estimation for discrete-time systems in the presence of timecorrelated disturbances and uncertain observations," IEEE Transactions on Automatic Control, vol. 39, no. 8, pp. 1636-1638, 1994.

[11] C. Sánchez-González and T. M. García-Muñoz, "Linear estimation for discrete systems with uncertain observations: an application to the correction of declared incomes in inquiry," Applied Mathematics and Computation, vol. 156, no. 1, pp. 211-233, 2004.

[12] W. Zhang, Y. Huang, and L. Xie, "Infinite horizon stochastic $H_{2} / H_{\infty}$ control for discrete-time systems with state and disturbance dependent noise," Automatica, vol. 44, no. 9, pp. 2306-2316, 2008.

[13] M. Sahebsara, T. Chen, and S. L. Shah, "Optimal $\mathrm{H}_{2}$ filtering with random sensor delay, multiple packet dropout and uncertain observations," International Journal of Control, vol. 80, no. 2, pp. 292 301, 2007.

[14] S. Nakamori, "Estimation technique using covariance information with uncertain observations in linear discrete-time systems," Signal Processing, vol. 58, no. 3, pp. 309-317, 1997.

[15] A. P. Sage and J. L. Melsa, Estimation Theory with Applications to Communications and Control, McGrawHill Series in Systems Science, McGraw-Hill, New York, NY, USA, 1971. 


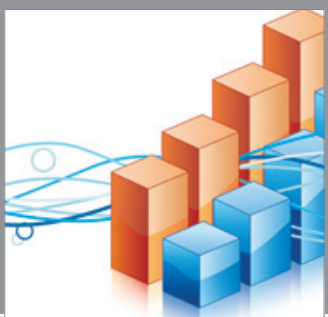

Advances in

Operations Research

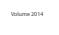

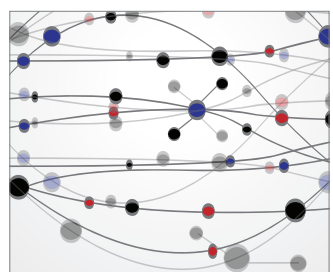

\section{The Scientific} World Journal
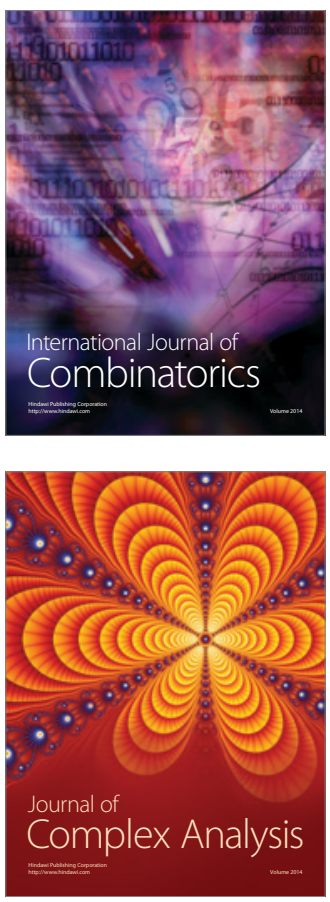

International Journal of

Mathematics and

Mathematical

Sciences
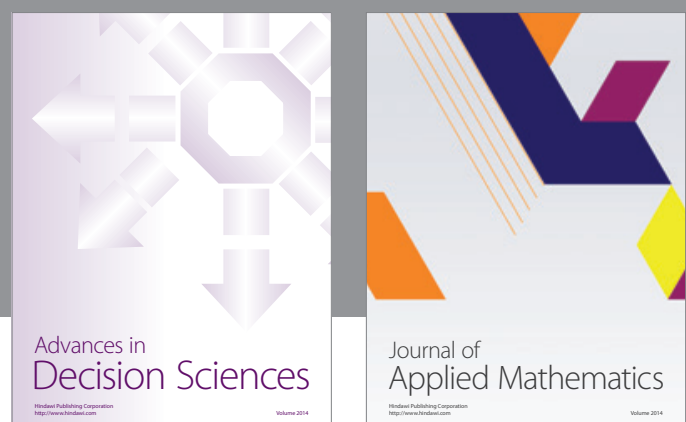

Journal of

Applied Mathematics
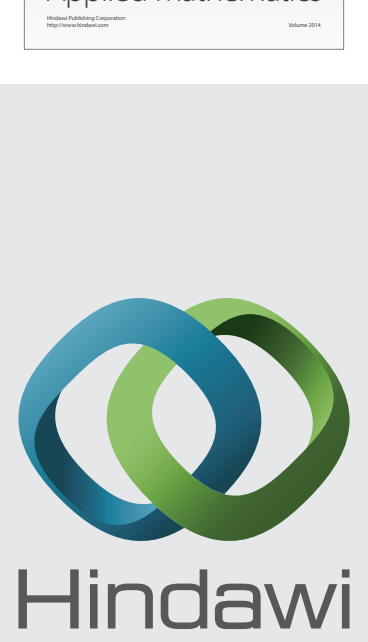

Submit your manuscripts at http://www.hindawi.com
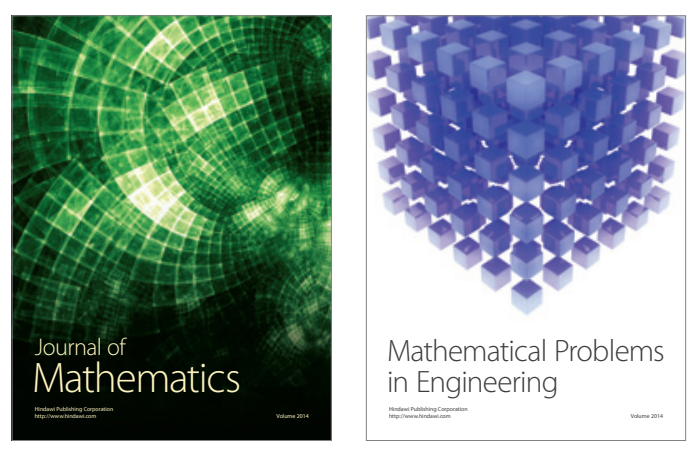

Mathematical Problems in Engineering
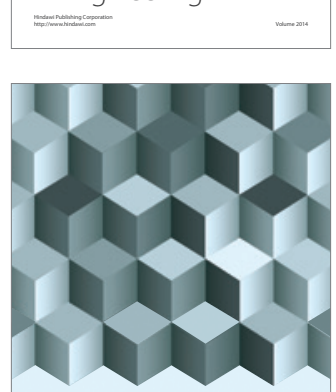

Journal of

Function Spaces
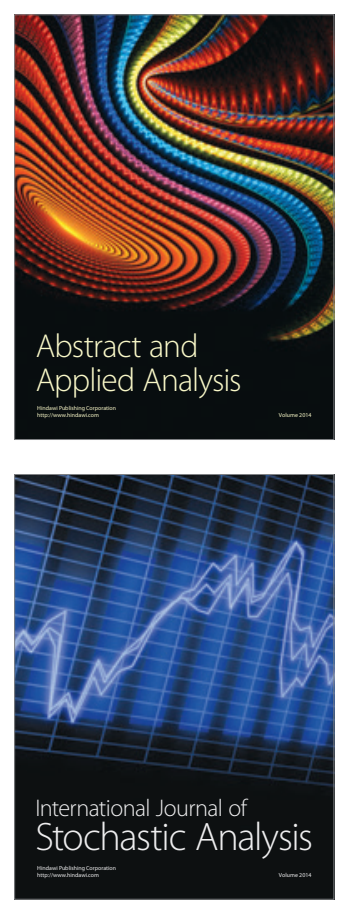

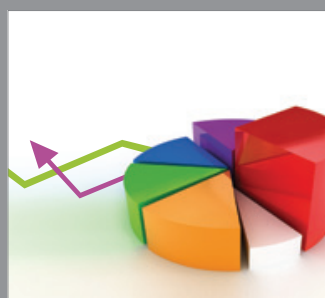

ournal of

Probability and Statistics

Promensencen
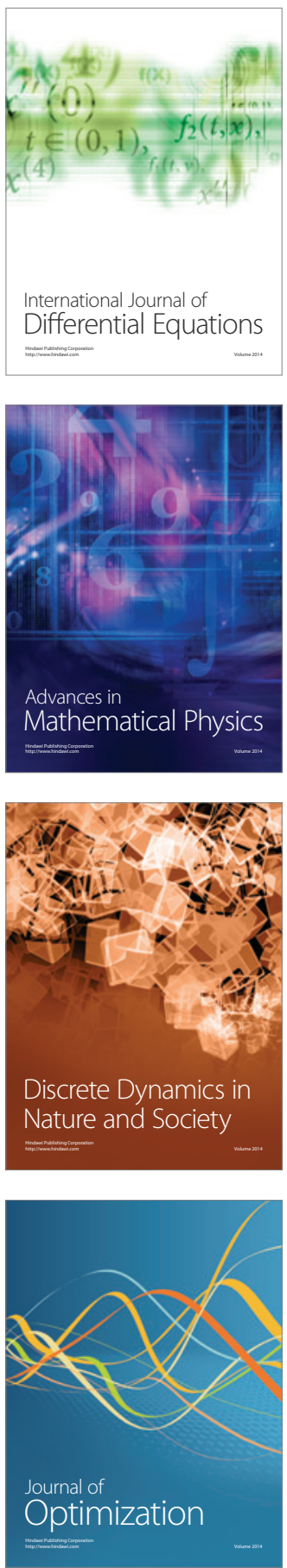